\title{
Stage-specific methylome screen identifies that NEFL is downregulated by promoter hypermethylation in breast cancer
}

\author{
SEONGEUN KANG ${ }^{1}$, BYUNGTAK KIM ${ }^{1}$, SUNG-BIN PARK ${ }^{1}$, GOOKJOO JEONG $^{1}$, \\ HAN-SUNG KANG ${ }^{2}$, RAN LIU ${ }^{3}$ and SUN JUNG KIM ${ }^{1}$ \\ ${ }^{1}$ Department of Life Science, Dongguk University-Seoul, Seoul 100-715; ${ }^{2}$ Research Institute and Hospital, \\ National Cancer Center, Gyeonggi do 411-764, Republic of Korea; ${ }^{3}$ Key Laboratory of Environmental \\ Medicine Engineering, Ministry of Education, School of Public Health, \\ Southeast University, Nanjing 210009, P.R. China
}

Received July 9, 2013; Accepted August 26, 2013

DOI: 10.3892/ijo.2013.2094

\begin{abstract}
Aberrant hypermethylation of promoter regions in specific genes is a key event in the formation and progression of breast cancers, and an increasing number of marker genes have been identified. However, few genes which show methylation change in accordance with the progression of breast cancer have been identified. To identify genes which consistently undergo promoter methylation alterations as the tumor develops from a benign to a malignant form, genome-wide methylation databases of breast cancer cell lines from stage I to stage IV were analyzed. Heatmap and cluster analysis revealed that the genome-wide methylation changes showed a good accordance with tumor progression. Seven out of 14,495 genes were found to be consistently increased alongside the promoter methylation level through the normal cell line to the cancer stage IV cell lines. NEFL, one of the in silico hypermethylated genes in cancer, showed hypermethylation and lower expression in the cancer cell line MDA-MB-231, as well as in cancer tissues (methylation, $\mathrm{p}<0.05$; expression, $\mathrm{p}<0.01$ ). The expression was restored by inducing demethylation of the promoter in MDA-MB-231
\end{abstract}

Correspondence to: Professor Sun Jung Kim, Department of Life Science, Dongguk University, 26 Pildong, Junggu, Seoul 100-715, Republic of Korea

E-mail: sunjungk@dongguk.edu

Dr Ran Liu, School of Public Health, Southeast University, Nanjing 210009, P.R. China

E-mail: ranliu@seu.edu.cn

Abbreviations: 5-Aza-dC, 5-aza-2'-deoxycytidine; CpG, cytosine guanine dinucleotide; ER, estrogen receptor; MSP, methylationspecific PCR; RT-PCR, reverse transcription-polymerase chain reaction

Key words: 5-aza-2'-deoxycytidine, breast cancer, cancer stage, genome-wide methylation, NEFL cells. Our findings may lend credence to the possibility of using tumor stage-specific alterations in methylation patterns as biomarkers for estimating prognosis and assessing treatment options for breast cancer.

\section{Introduction}

Carcinogenesis of breast cancer is a complicated process that is initiated from a limited number of cancer cells and proceeds to malignancy (1). Genetic as well as epigenetic changes of tumor suppressors and/or oncogenes have been known to contribute to the process (2). Promoter methylation change leads to epigenetic modification which also includes ones by histone change and miRNA $(3,4)$. Well known tumor suppressors, such as p53, RASSF1A and BRCA1, are inactivated by hypermethylation of their promoters in breast cancer (5-7). So far, examination of methylation has been focused on comparison of the methylation level between normal and cancer cells, or before and after the administration of chemical treatments to cultured cells and tissue. Genome-wide methylation screen in normal vs. tumor tissue identified epigenetically modified biomarkers, including BCAB, HOXD1 and POU4F1, revealing an over-representation of homeobox genes and genes involved in the regulation of transcription (8). It has been further claimed that aberrant methylation occurs at an early stage in breast tumorigenesis $(9,10)$. In accordance with this hypothesis, TLX1, HOXB13 and Caveolin-1 showed early onset of methylation in stage I breast tumors (11).

Methylation change can be associated with tumor stage or site of tumor (12). For example, trends of increasing RASSF1 methylation were observed to occur in association with increasing tumor size and advancement of the stage of breast cancer (13). In another study, ADAM33 was determined to be silenced by promoter methylation, showing hypermethylation in invasive lobular carcinoma (76.2\%) compared to invasive ductal carcinoma (25.5\%) (14).

Even though the number of genes which are associated with methylation change in carcinogenesis are being increased, the methylation trend has been observed in only a few cases. A 
functional hypermethylome screen identified genes such as CKM and TAC1 which show a stage-dependent methylation frequency pattern, as candidates to help delineate breast cancer prognostic signatures (15). An in vitro model of human breast epithelial cell transformation with MCF-10F normal cell line and its transformed derivatives at transformed stage, invasive stage, and tumor stage showed increased DNA methylation during the invasive and tumor stages (16). In the study, NRG1 was unmethylated in normal cells and transformed cells, becoming hypermethylated in the invasive and tumor stage. Few systemic trials have been carried out in a way which identifies genes showing linearity between promoter methylation and tumor progression (17).

In this study, we analyzed the Illumina methylation array data that were registered on the GEO database, representing genome-wide methylation at a normal breast cell line and at tumor cell lines of cancer stages I-IV. Comparison analysis revealed a close relationship between the genome-wide methylation and cancer stage. In addition, NEFL was identified as a novel epigenetic marker, which was hypermethylated and downregulated in breast cancer.

\section{Materials and methods}

Study subjects. All patients provided written informed consent to donate removed tissue to the National Cancer Center (NCC, Seoul) in Korea and samples were obtained according to protocols approved by the Research Ethics Board of NCC. Forty pairs of breast cancer (BrCa) and their corresponding adjacent normal tissue specimens were obtained from patients who had undergone surgery between 2011 and 2012 at NCC. $\mathrm{BrCa}$ specimens were subjected to histological examination by an expert pathologist for independent confirmation of the cancer stage.

Cell culture. A normal breast cell line, MCF 10A, and breast cancer cell lines, HCC1395 (stage I) and MDA-MB-231 (stage IV) were purchased from American Type Culture Collection (ATCC; Manassas, VA). HCC38 (stage II) was purchased from Korea Cell Line Bank (KCLB; Seoul, Korea). HCC38, HCC1395 and MDA-MB-231 were cultured in RPMI-1640 supplemented with $10 \%$ fetal bovine serum (FBS). MCF 10A was grown in MEBM supplemented with MEGM Single Quots (Lonza, Basel, Switzerland). All cell types were cultured on the surface of a $75 \mathrm{~cm}^{2}$ culture flask.

Methylation-specific PCR (MSP). Chromosomal DNA from approximately $100 \mathrm{mg}$ of tissue samples was isolated using a genomic DNA purification kit (Promega, Madison, WI) according to the manufacturer's protocol, with a $60 \mu \mathrm{l}$ elution volume. Chromosomal DNA from cultures in a $75 \mathrm{~cm}^{2}$ flask was isolated using an AllPrep DNA/RNA Mini Kit (Qiagen, Valencia, CA) with $100 \mu$ l elution volume. Sodium bisulfite modification of genomic DNA and PCR was carried out as previously described (18). Briefly, $0.1 \mathrm{mg}$ of DNA was treated with sodium bisulfite and then PCR was carried out using primers (Table I) and a Kapa SYBR Fast qPCR kit (Kapa Biosystems, Woburn, MA). A methylation index was calculated for each sample using the following formula: methylation index $=1 /\left[1+2^{-(\mathrm{CTu}-\mathrm{CTme})}\right] \times 100 \%$, where $\mathrm{CTu}$ is the cycle threshold (CT) obtained using the unmethylated primer pair and CTme is the average CT obtained using the methylated primer pair.

Real-time RT-PCR. Total RNA from approximately $100 \mathrm{mg}$ of tissues was prepared using TRIzol reagent according to the manufacturer's protocols (Gibco-BRL, Carlsbad, CA), and was finally suspended in $30 \mu \mathrm{l}$ of distilled water. Total RNA from a $75 \mathrm{~cm}^{2}$ culture flask was prepared using the RNeasy Plus mini kit (Qiagen) and was finally eluted with $30 \mu \mathrm{l}$ of distilled water. Reverse transcription was conducted using $5 \mu \mathrm{g}$ of total RNA with a reverse transcription kit (Promega). Expression levels of selected genes were measured by real-time quantitative RT-PCR analysis using a Kapa SYBR Fast qPCR kit (Kapa Biosystems) on an ABI 7300 instrument (Applied Biosystems). One microliter of cDNA was used for PCR, which was performed in duplicate. Primers used for the RT-PCR are listed in Table I. RNA quantity was normalized to GAPDH content, and gene expression was quantified according to the $2^{-\Delta \mathrm{Ct}}$ method.

Databases and statistical analysis. The Infinium Methylation Chip data for a breast normal cell line and cancer cell lines were obtained from the GEO database (http://www.ncbi.nlm. nih.gov/geo/). The selected cell lines are: normal, MCF 10A; breast cancer stage I, HCC1395; stage II, HCC1008, HCC1143, HCC1954, HCC1937, HCC38; stage III, HCC1599, HCC2218; stage IV, HCC1569, MDA-MB-231. Observations with adjusted $p$-values $\geq 0.05$ were removed, and thus excluded from further analysis. Following adjustment, the remaining genes were defined as differentially methylated if they displayed increased methylation levels when compared to that of the previous stage of the cell line. A hierarchical clustering dendrogram was generated by Gene Cluster 3.0 (http://bonsai. hgc.jp/ mdehoon/software/cluster/software.htm) to determine the relationship between the methylation profile and developmental stage of the tumor. Student's t-test was used to detect differences in mean levels of methylation and the expression level between the normal and cancer tissues using SPSS for Windows, version 17.0 (SPSS Inc., Chicago, IL). P-values $<0.05$ were considered to be statistically significant.

\section{Results}

In silico methylome analysis revealed that genome-wide methylation is closely linked with cancer development. A group of genome-wide methylation databases was collected and analyzed for genes for which the methylation level consistently increased or decreased with the progress of breast tumor stage. The data were obtained by carrying out Illumina methylation array covering 14,495 genes and 27,578 CpG sites, and databases were available for cell lines and tissues at the GEO database (http://www.ncbi.nlm.nih.gov/ $\mathrm{geo} /$ ). As it is well known that the estrogen receptor (ER) status of cancer cells affects the methylation and expression of many genes in breast cancer $(19,20)$, databases were first screened so that they were derived from the same ER status. Of the databases, only ER-negative (ER-) cell lines were available with a full set, which included at least one database at both normal and all the cancer developmental 
Table I. Sequences of primers employed in this study.

\begin{tabular}{lcc}
\hline Genes & \multicolumn{1}{c}{ Forward primer $\left(5^{\prime} \rightarrow 3^{\prime}\right)$} & Reverse primer $\left(5^{\prime} \rightarrow 3^{\prime}\right)$ \\
\hline MSP & & \\
NEFL M & TTGTTGAGGTAGTCGCGcgT & AAATCAACCAACTACAAAACTTAT \\
NEFL U & TTGTTGAGGTAGTTGTGtgT & AAATCAACCAACTACAAAACTTAT \\
Real-time RT-PCR & & \\
NEFL & CAAAGAGTGAAATGGCACGAT & TCCAAGAGTTTCCTGCCTGT \\
GAPDH & CAGGAGGCATTGCTGATGAT & GAAGGCTGGGGCTCATTT
\end{tabular}

Table II. Genes displaying consistently increasing methylation pattern according to cancer development in breast cancer cell lines

\begin{tabular}{|c|c|c|c|c|c|c|c|}
\hline \multirow[b]{2}{*}{ Gene } & \multirow[b]{2}{*}{ Accession no. } & \multirow[b]{2}{*}{ Description } & \multicolumn{5}{|c|}{ Stage } \\
\hline & & & Normal & I & II & III & IV \\
\hline NEFL & NM_006158.1 & $\begin{array}{l}\text { Neurofilament; } \\
\text { light polypeptide } 68 \mathrm{kDa}\end{array}$ & $0.04^{\mathrm{a}}$ & 0.05 & 0.18 & 0.56 & 0.86 \\
\hline PPP1R14C & NM_030949.1 & $\begin{array}{l}\text { Protein phosphatase } 1 \text {; } \\
\text { regulatory (inhibitor) subunit } 14 \mathrm{C} \text {; } \\
\text { serologically defined breast cancer } \\
\text { antigen NY-BR-81 }\end{array}$ & 0.03 & 0.03 & 0.27 & 0.56 & 0.74 \\
\hline TEC & NM_003215.1 & Tec protein tyrosine kinase & 0.59 & 0.60 & 0.62 & 0.68 & 0.81 \\
\hline PFDN1 & NM_002622.3 & Prefoldin 1 & 0.01 & 0.01 & 0.03 & 0.03 & 0.07 \\
\hline MSRA & NM_012331.2 & Methionine sulfoxide reductase A & 0.01 & 0.02 & 0.04 & 0.03 & 0.13 \\
\hline TTLL3 & NM_015644.2 & $\begin{array}{l}\text { Tubulin tyrosine ligase-like family; } \\
\text { tubulin-tyrosine ligase activity }\end{array}$ & 0.02 & 0.11 & 0.42 & 0.48 & 0.78 \\
\hline KIAA0372 & NM_014639.2 & $\begin{array}{l}\text { Hypothetical protein LOC } 9652 \text {; } \\
\text { structural constituent of ribosome }\end{array}$ & 0.07 & 0.08 & 0.17 & 0.13 & 0.33 \\
\hline
\end{tabular}

${ }^{a}$ Methylation level ( $\beta$-value) in normal and cancer cell lines. The roman numbers denote specific cancer stage of the cell lines. The values are average of cell lines at each stage.

stages, I-IV. Therefore an ER - normal cell line, MCF 10A, and ten $\mathrm{ER}^{-}$cancer cell lines were selected and analyzed. To examine the correlation between the methylation change and tumor progression, the genes from the microarray were first filtered so that false-negative genes $(\mathrm{p}<0.05)$ were excluded. In total, $12,787 \mathrm{CpG}$ sites remained common to all normal and cancer cells. Class comparison and heatmap analysis were carried out based on the methylation index of the genes. Interestingly, the cell lines were arranged in the dendrogram in such a way that normal cells and early stage cells were linked closer together, and likewise, late stage cells were located closer to each other (Fig. 1). Notably, the two cell lines at stage IV, HCC1569 and MDA-MB-231, were most closely related, showing the shortest distance. This result indicates that the genome-wide methylation change from benign to advanced cancerous cell occurs with a trend during carcinogenesis.

Identification of hypermethylated genes with consistent change of methylation according to cancer stages. Genes showing a gradual increase or decrease alongside tumor development were identified by comparing their methylation levels after screening statistically significant genes (Table II). First,

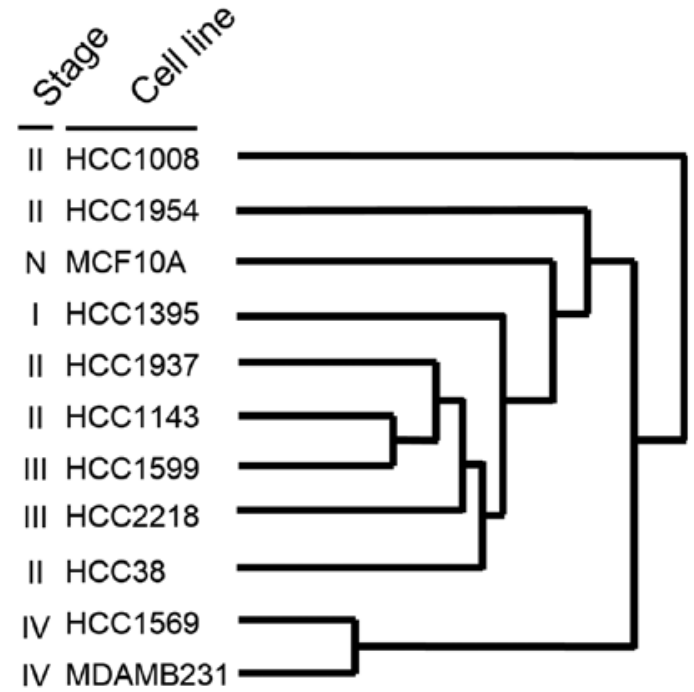

Figure 1. Cluster analysis of breast cell lines with genes of altered methylation through developmental stages of breast cancer cell lines. Analysis was carried out using a methylation index ( $\beta$-value) taken from Illumina Human Methylation 27 Bead Chip database registered at the GEO. Hierarchical clustering with the correlation distance was shown for a normal breast cell line and tumor cell lines at various stages (stages I-IV). 
A
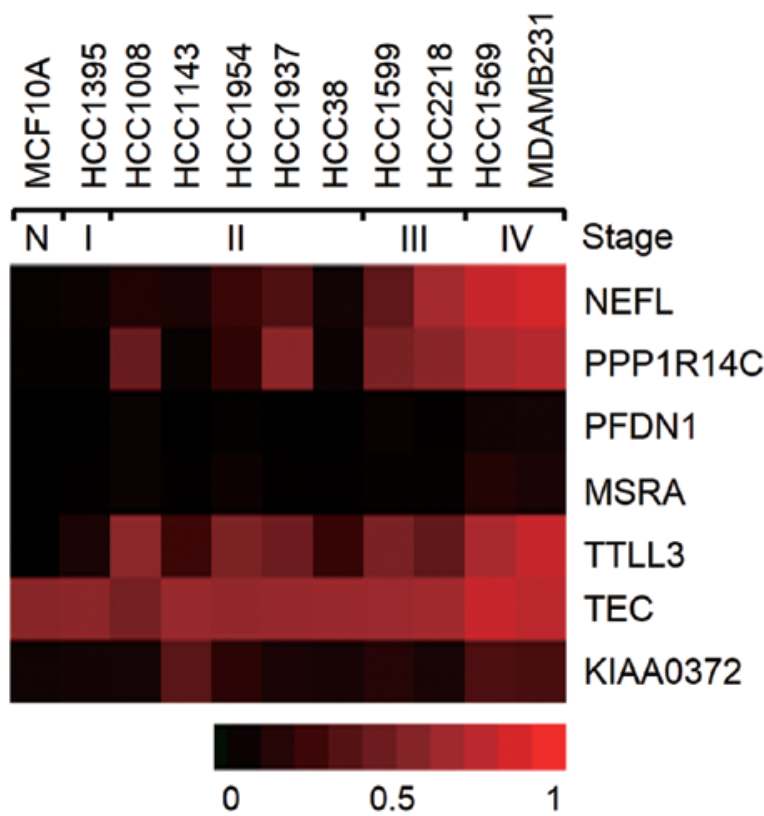

B

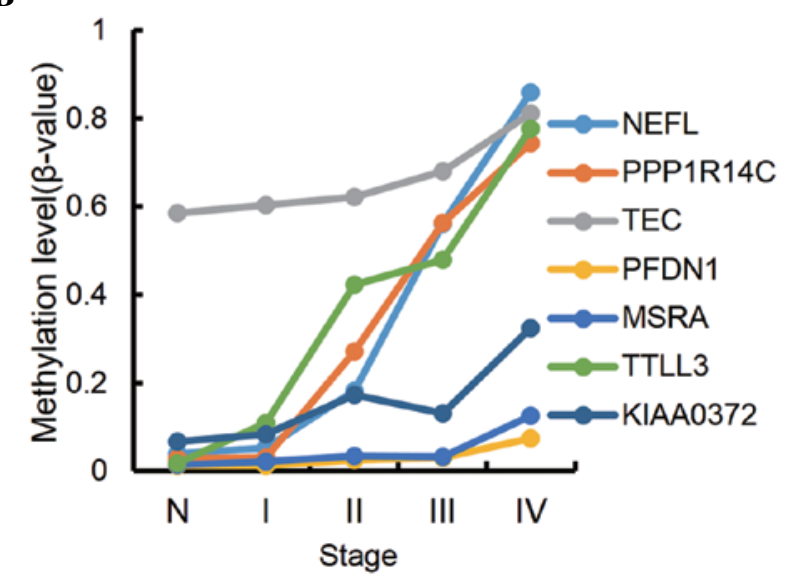

Figure 2. Selected genes showing consistent increases of methylation along with cancer stage in breast cancer cells. (A) A heatmap diagram of genes with consistent increases of methylation level in accordance with cancer stage progression. (B) Hypermethylated genes with a consistent increase of methylation level. The methylation values were taken from the average of the examined cell lines.

observations with adjusted $\mathrm{p}$-values $\geq 0.05$ were removed, and thus excluded from further analysis. Then, using an MS Excel-based filtering procedure, genes of which methylation level consistently increases or decreases as the tumor cell lines develops into the higher cancer stage were selected. As shown in Fig. 2, seven genes were revealed to be consistently hypermethylated. It is notable that NEFL, PPP1R14C and TTLL3 showed a step-wise increase of methylation through all the stage from $3.0-17 \%$ methylation at stage I to $74-86 \%$ methylation at stage IV. For the hypomethylated genes in cancer, no remarkable genes were found, which showed a consistent decrease of methylation from normal cells to stage IV cancer cells (data not shown). PPP1R14C is a protein phosphatase 1 inhibitor, and has been previously identified as an upregulating gene after induction of demethylation in melanoma cell lines (21). TTLL3 is a tubulin glycine ligase which regulates the assembly of microtubules, of which the relevance with cancer has yet to be elucidated (22).
NEFL is downregulated by hypermethylation in breast cancer. To reveal the effect of methylation on gene expression for the in silico identified genes, and thereby to develop potential epigenetic breast cancer markers, NEFL, which has shown a consistent increase of methylation, was selected and examined for its promoter methylation and expression in both cell lines and tissues. NEFL is a component of the three-subunit protein, neuronal intermediate filament (23). In addition to its influence on the nervous system, NEFL has been shown to act as a tumor suppressor in several types of cancer (24). However, the epigenetic mechanism of the downregulation has not been elucidated.

It was found that NEFL was downregulated in all examined cancer cell lines of stages I, II, and IV, compared to the normal cell line (Fig. 3B). Induction of demethylation by 5-Aza-dC in the MDA-MB-231 cells recovered the expression with a 4-fold increase (Fig. 3D). Next, NEFL was examined for its methylation and expression in breast cancer tissues. Thirty-six pairs of cancer tissue and normal tissues from the same area were examined for promoter methylation by MSP analysis. The result indicated that the gene is hypermethylated in cancer tissues compared to normal tissues $(\mathrm{p}<0.05)$ (Fig. 4). Real-time RT-PCR was carried out to monitor NEFL expression and the result indicated that the gene is downregulated in cancer tissues $(\mathrm{p}<0.01)$ (Fig. 5). These molecular experimental results together with the in silico data suggest that NEFL could be developed as an epigenetic marker for breast cancer.

\section{Discussion}

This study was carried out with the aim of identifying breast cancer stage-specific markers which are regulated by promoter methylation, and which show a consistent increase or decrease of methylation level during carcinogenesis. Cluster analysis determined that there was a significant correlation between genome-wide methylation and cancer stage, possibly implying that genome-wide methylation at each cancer stage poses its unique pattern and also changes following the cancer stages.

In terms of DNA methylation, cancer cells show global hypomethylation and site-specific promoter hypermethylation $(25,26)$. When the methylation level of the 26,250 filtered genes from the database were summed up, there was no significant difference between the normal cell lines and various cancer stages. Furthermore, only a few genes showed a consistent increase of methylation as the cancer cell line stage increased. These facts may indicate that the genome-wide methylation at the promoters undergo dynamic hypermethylation as well as hypomethylation depending on specific genes. However, it should be mentioned that the data from the database did not cover the whole $\mathrm{CpG}$ sites of the genome.

Genome-wide analysis identified candidate genes showing a consistently increasing methylation pattern. It still remains controversial when cancer-related genes are methylated during carcinogenesis. Early onset of methylation helps make DNA methylation biomarkers attractive predictors for the development of effective diagnostic tests for the early detection of neoplasia (27). The late stage onset of methylation may be difficult to detect at the early stages of carcinogenesis. Nonetheless, there appeared gene sets which showed the highest methylation at a specific cancer stage. These results imply that the specific cancer stage is predictable using the 

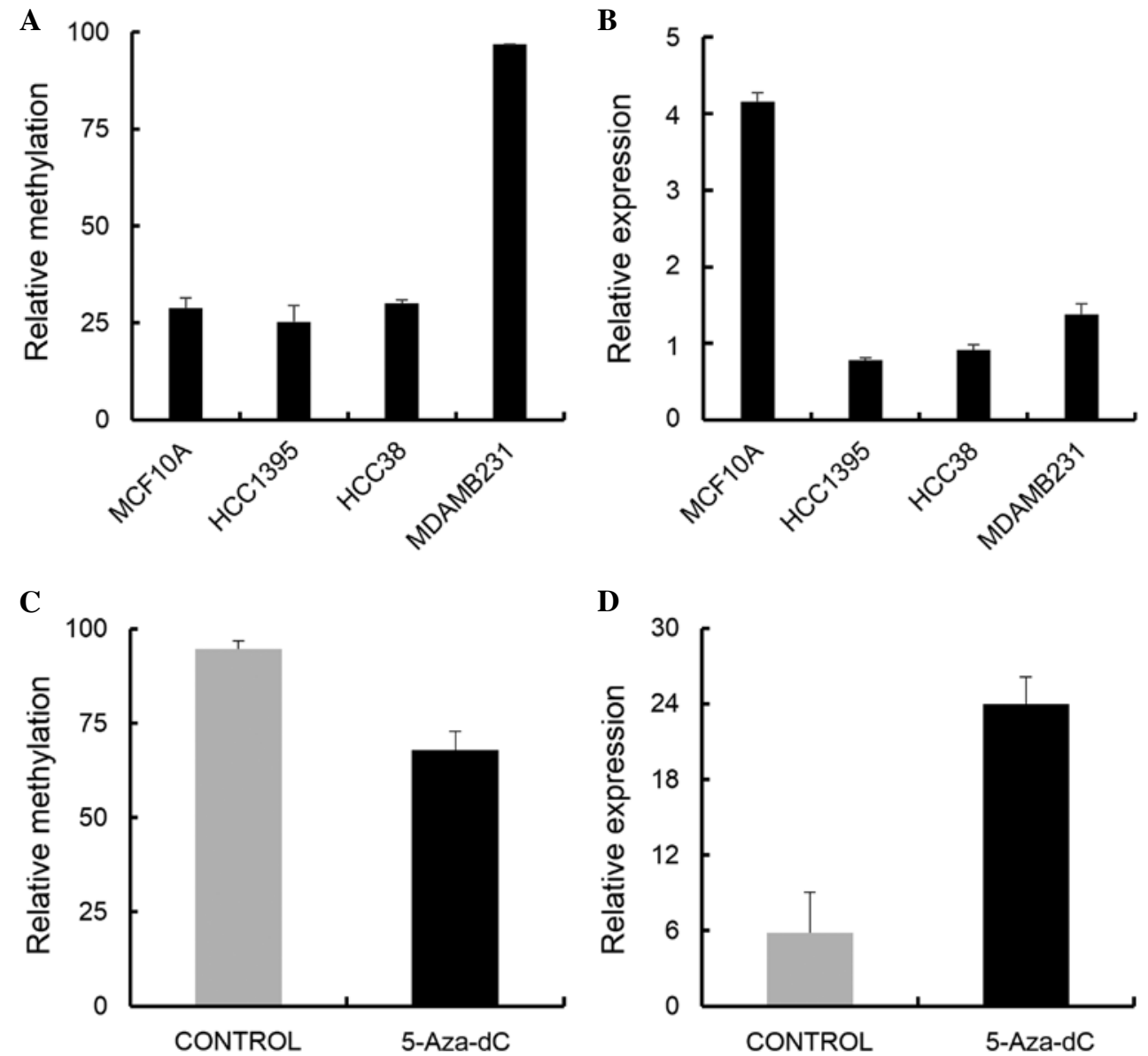

Figure 3. NEFL is hypermethylated and downregulated in the late stage of breast cancer cell line. (A) Real-time MSP and (B) real-time RT-PCR analysis of NEFL were performed in the normal cell line, MCF 10A, and the cancer cell lines, HCC38, HCC1395 and MDA-MB-231. (C) Real-time MSP and (D) real-time RT-PCR analysis of NEFL were performed after the cancer cell culture MDA-MB-231 was treated with 5-Aza-dC. Each sample was examined in duplicate for three independent experiments, and the average relative methylation and expression levels are presented with standard errors.

stage-specific methylation markers. To do this, however, the methylation data for candidate genes should be accumulated from a larger collection of cancer tissues.

In previous studies, loss of heterozygosity pattern on the human chromosomal region 8p12-p21 harboring the NEFL gene was frequently observed in breast carcinoma, suggesting its role as a tumor suppressor (28). This is in accordance with another study where NEFL expression was downregulated in malignant breast cancer (24). The relationship between promoter methylation and expression is known only in head and neck cancer (29). In the study, NEFL was identified as a hypermethylated gene associated with resistance to cisplatinbased chemotherapy in head and neck cancer. However, no analysis of the relationship between promoter methylation and expression has been carried out in cancer.

The database adopted in this study contains only methylation data from ER cancer cell lines due to the incompleteness of $\mathrm{ER}^{+}$cell lines, and due to the lack of ER status information for cancer tissues. It is known that ER affects the promoter methylation as well as the expression of specific genes. In general, ER-positive tumors displayed more hypermethylated loci than ER-negative tumors. However, the hypermethylated loci in ER-negative tumors were clustered closer to the transcriptional start site compared with ER-positive tumors
(20). At the gene level, $\mathrm{ER}^{+}$and $\mathrm{ER}^{-}$cancer displayed different sets of hypermethylated or hypomethylated genes. MGMT, involved in direct DNA repair, was frequently hypermethylated in the triple-negative (ER/PgR/Her3 negative) breast cancer (30). Meanwhile, FAM124B and ST6GALNAC1 were hypermethylated in $\mathrm{ER}^{+} / \mathrm{PR}^{+}$breast cancer (31). In addition, methylation of miRNAs also showed association with ER status. The promoter of the hsa-mir-200b cluster is hypermethylated in breast cancer and its methylation is associated with the loss of either estrogen receptor or progesterone receptor (19).

Taken together, we found that genome-wide methylation status is closely related with the specific stage of breast cancer development, implying a gradual change of global methylation during carcinogenesis. In addition, a subset of genes showed a gradual increase or decrease of methylation in accordance with breast cancer development in cell lines with a sudden change from stage II to stage IV. NEFL, identified in silico as a gene showing a gradual increase of methylation level, was proven to be upregulated in breast cancer tissue as well as cell lines. Further investigation into the mechanisms leading to differential methylation status with breast tumor stage and progression may provide additional insights, which could prove useful in estimating prognosis and determining treatment options. 


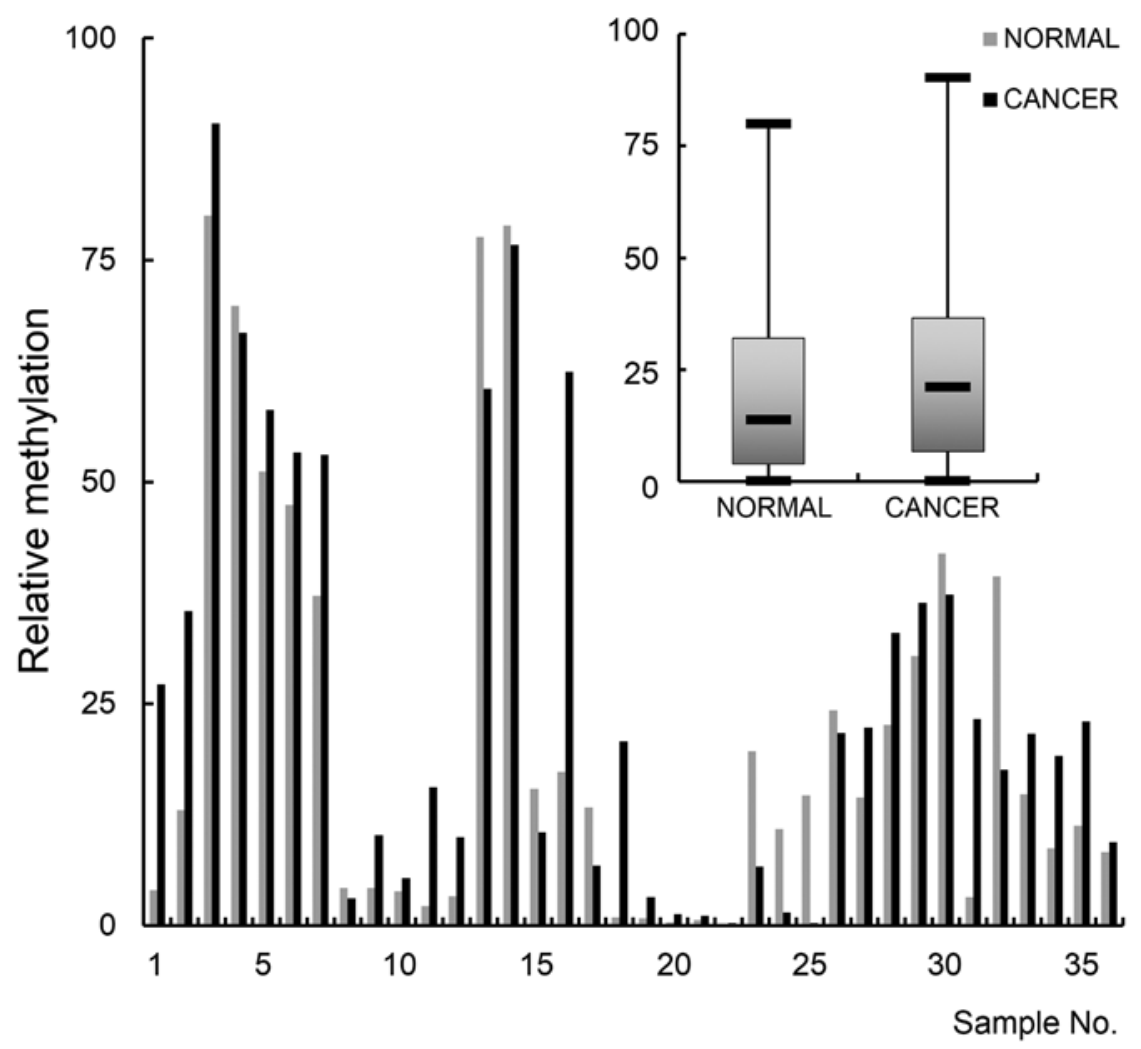

Figure 4. NEFL is hypermethylated in breast cancer tissues. Methylation levels of the CpGs at the promoter were examined by MSP for 36 pairs of tumor tissues and their surrounding normal tissues. Each sample was examined in duplicate and the average relative methylation and expression levels are presented. Insets are box plots of the same data which appears in the bar graph.

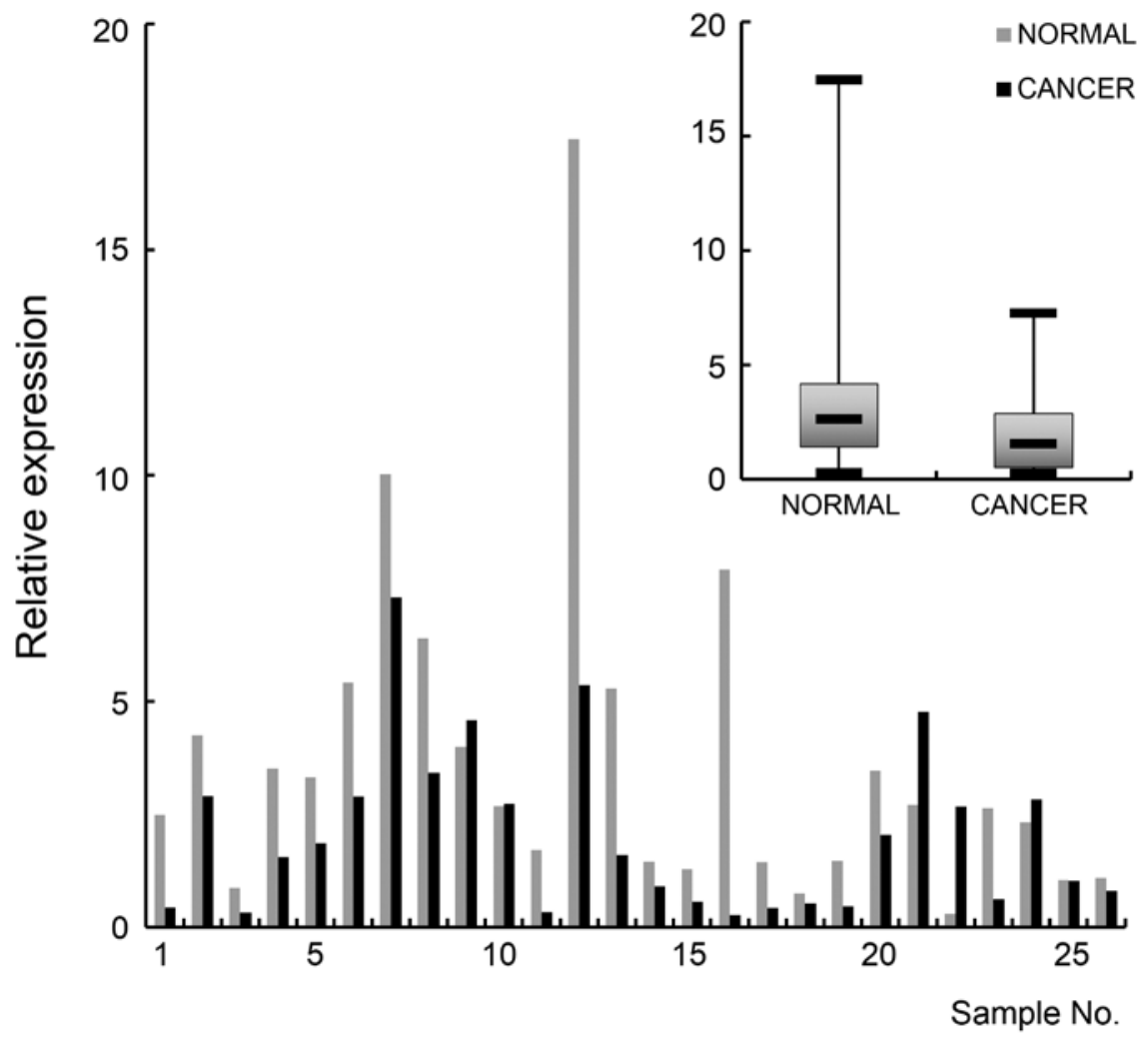

Figure 5. NEFL is downregulated in breast cancer tissues. The expression level of NEFL was examined using real-time RT-PCR for 26 pairs of tumor tissues and their surrounding normal tissues. Each sample was examined in duplicate and the average relative methylation and expression levels are presented. Insets are box plots of the same data which appears in the bar graph. 


\section{Acknowledgements}

The authors thank S.H. Kang for his contribution to preparation of the manuscript. This study was supported by the Basic Science Research Program (NRF-2012R1A1A2040830) and Korea-China Joint Program (NRF-2011-616-C00056) through the National Research Foundation of Korea (NRF), funded by the Ministry of Education, Science and Technology, and by a National Science Foundation of China (NSFC) Grant (nos. 81172747 and 81111140396 ).

\section{References}

1. Pare R, Yang T, Shin JS and Lee CS: The significance of the senescence pathway in breast cancer progression. J Clin Pathol 66: 491-495, 2013

2. Harburg GC and Hinck L: Navigating breast cancer: axon guidance molecules as breast cancer tumor suppressors and oncogenes. J Mammary Gland Biol Neoplasia 16: 257-270, 2011.

3. Shi L, Sun L, Li Q, et al: Histone demethylase JMJD2B coordinates $\mathrm{H} 3 \mathrm{~K} 4 / \mathrm{H} 3 \mathrm{~K} 9$ methylation and promotes hormonally responsive breast carcinogenesis. Proc Natl Acad Sci USA 108: 7541-7546, 2011.

4. Krell J, Frampton AE, Jacob J, Castellano L and Stebbing J: miRNAs in breast cancer: ready for real time? Pharmacogenomics 13: 709-719, 2012

5. Kang JH, Kim SJ, Noh DY, et al: Methylation in the p53 promoter is a supplementary route to breast carcinogenesis: correlation between $\mathrm{CpG}$ methylation in the $\mathrm{p} 53$ promoter and the mutation of the p53 gene in the progression from ductal carcinoma in situ to invasive ductal carcinoma. Lab Invest 81 573-579, 2001

6. Yan PS, Shi H, Rahmatpanah F, et al: Differential distribution of DNA methylation within the RASSF1A CpG island in breast cancer. Cancer Res 63: 6178-6186, 2003.

7. Matros E, Wang ZC, Lodeiro G, Miron A, Iglehart JD and Richardson AL: BRCA1 promoter methylation in sporadic breast tumors: relationship to gene expression profiles. Breast Cancer Res Treat 91: 179-186, 2005.

8. Faryna M, Konermann C, Aulmann S, et al: Genome-wide methylation screen in low-grade breast cancer identifies novel epigenetically altered genes as potential biomarkers for tumor diagnosis. FASEB J 26: 4937-4950, 2012.

9. Van Hoesel AQ, Sato Y, Elashoff DA, et al: Assessment of DNA methylation status in early stages of breast cancer development. Br J Cancer 108: 2033-2038, 2013.

10. Miyamoto K, Fukutomi T, Akashi-Tanaka S, et al: Identification of 20 genes aberrantly methylated in human breast cancers. Int J Cancer 116: 407-414, 2005.

11. Chen ST, Lin SY, Yeh KT, et al: Mutational, epigenetic and expressional analyses of caveolin-1 gene in breast cancers. Int J Mol Med 14: 577-582, 2004

12. Hu M, Yao J, Cai L, et al: Distinct epigenetic changes in the stromal cells of breast cancers. Nat Genet 37: 899-905, 2005.

13. Sebova K, Zmetakova I, Bella V, et al: RASSF1A and CDH1 hypermethylation as potential epimarkers in breast cancer. Cancer Biomark 10: 13-26, 2011.
14. Mohammad J, Zeerak A and Hjerten S: Dye-ligand affinity chromatography on continuous beds. Biomed Chromatogr 9: 80-84, 1995.

15. Jeschke J, Van Neste L, Glockner SC, et al: Biomarkers for detection and prognosis of breast cancer identified by a functional hypermethylome screen. Epigenetics 7: 701-709, 2012.

16. Fernandez SV, Snider KE, Wu YZ, Russo IH, Plass C and Russo J: DNA methylation changes in a human cell model of breast cancer progression. Mutat Res 688: 28-35, 2010.

17. Loss LA, Sadanandam A, Durinck S, et al: Prediction of epigenetically regulated genes in breast cancer cell lines. BMC Bioinformatics 11: 305, 2010.

18. Kim JH, Kang HS, Kim TW and Kim SJ: Differential methylation hybridization profiling identifies involvement of STAT1-mediated pathways in breast cancer. Int J Oncol 39: 955-963, 2011.

19. Wee EJ, Peters K, Nair SS, et al: Mapping the regulatory sequences controlling 93 breast cancer-associated miRNA genes leads to the identification of two functional promoters of the Hsa-mir-200b cluster, methylation of which is associated with metastasis or hormone receptor status in advanced breast cancer. Oncogene 31: 4182-4195, 2012.

20. Fackler MJ, Umbricht CB, Williams D, et al: Genome-wide methylation analysis identifies genes specific to breast cancer hormone receptor status and risk of recurrence. Cancer Res 71: 6195-6207, 2011.

21. Bonazzi VF, Irwin D and Hayward NK: Identification of candidate tumor suppressor genes inactivated by promoter methylation in melanoma. Genes Chromosomes Cancer 48: 10-21, 2009.

22. Wloga D, Webster DM, Rogowski K, et al: TTLL3 is a tubulin glycine ligase that regulates the assembly of cilia. Dev Cell 16: 867-876, 2009

23. Abe A, Numakura C, Saito K, et al: Neurofilament light chain polypeptide gene mutations in Charcot-Marie-Tooth disease: nonsense mutation probably causes a recessive phenotype. J Hum Genet 54: 94-97, 2009.

24. Li XQ, Li L, Xiao CH and Feng YM: NEFL mRNA expression level is a prognostic factor for early-stage breast cancer patients. PLoS One 7: e31146, 2012.

25. Shen L, Kondo Y, Guo Y, et al: Genome-wide profiling of DNA methylation reveals a class of normally methylated $\mathrm{CpG}$ island promoters. PLoS Genet 3: 2023-2036, 2007.

26. Sincic N and Herceg Z: DNA methylation and cancer: ghosts and angels above the genes. Curr Opin Oncol 23: 69-76, 2011.

27. Carmona FJ and Esteller M: DNA methylation in early neoplasia. Cancer Biomark 9: 101-111, 2010.

28. Seitz S, Werner S, Fischer J, Nothnagel A, Schlag PM and Scherneck S: Refined deletion mapping in sporadic breast cancer at chromosomal region 8p12-p21 and association with clinicopathological parameters. Eur J Cancer 36: 1507-1513, 2000.

29. Chen B, Chen J, House MG, Cullen KJ, Nephew KP and Guo Z: Role of neurofilament light polypeptide in head and neck cancer chemoresistance. Mol Cancer Res 10: 305-315, 2012.

30. Fumagalli C, Pruneri G, Possanzini P, et al: Methylation of O6-methylguanine-DNA methyltransferase (MGMT) promoter gene in triple-negative breast cancer patients. Breast Cancer Res Treat 134: 131-137, 2012.

31. Li L, Lee KM, Han W, et al: Estrogen and progesterone receptor status affect genome-wide DNA methylation profile in breast cancer. Hum Mol Genet 19: 4273-4277, 2010. 